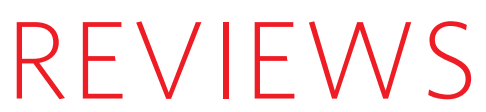

\title{
Towards a molecular understanding of shape selectivity
}

\author{
Berend Smit ${ }^{1,2,3} \uparrow \&$ Theo L. M. Maesen ${ }^{4} \dagger$
}

\begin{abstract}
Shape selectivity is a simple concept: the transformation of reactants into products depends on how the processed molecules fit the active site of the catalyst. Nature makes abundant use of this concept, in that enzymes usually process only very few molecules, which fit their active sites. Industry has also exploited shape selectivity in zeolite catalysis for almost 50 years, yet our mechanistic understanding remains rather limited. Here we review shape selectivity in zeolite catalysis, and argue that a simple thermodynamic analysis of the molecules adsorbed inside the zeolite pores can explain which products form and guide the identification of zeolite structures that are particularly suitable for desired catalytic applications.
\end{abstract}

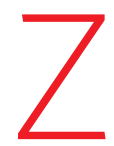
eolites are microporous mineral materials that have found wide use in industry since the late 1950s, with one of their most important applications being chemical catalysis. They are particularly important as cracking catalysts in oil refining. One of their defining features-apart from being solid catalysts that are easy to recycle-is that the shape, or topology, of the internal pore structure of a zeolite can strongly affect the selectivity with which particular product molecules are formed in chemical transformations catalysed by the zeolite. Here, we will argue that this shape selectivity can be explained by very simple thermodynamic analyses that consider the impact of zeolite topology on the free energy landscape; that is, on the free energies of formation of the various molecules involved in the catalysed reactions.

The analyses presented here are simple and straightforward, yet have become feasible only relatively recently as advances in molecular simulation techniques have started to provide access to the thermodynamic data underpinning them. After a short introduction of zeolites and their use as catalysts, we will therefore also briefly outline the developments in simulation capabilities that give access to the thermodynamic information crucial for our understanding of zeolite catalysis. We then show how the free-energy-landscape approach can elucidate the molecular-level mechanism(s), giving rise to shape selectivity in a number of simple yet industrially important processes. We conclude this review by outlining the crucial issues that need to be addressed to take the free-energy-landscape approach to the next stage, where the combined use of simulations and thermodynamic analysis might have profound implications for how we screen and develop zeolite-based catalysts.

\section{Zeolites as industrial catalysts}

Zeolites are crystalline aluminosilicates with a three-dimensional framework that consists of nanometre-sized channels and cages and imparts high porosity and a large surface area to the material. The basic structural unit of all zeolite frameworks consists of a silicon or aluminium atom tetrahedrally coordinated to four oxygen atoms. Any zeolite built of silica and oxygen only is neutral, but replacing $\mathrm{Si}^{4+}$ by $\mathrm{Al}^{3+}$ creates a negative charge on the framework. All such framework charges are neutralized by cations that reside inside the zeolite pores, where they can move freely and be exchanged against other cations. When protons neutralize framework charges, they constitute acid sites that can catalyse the two types of reaction important in all oil refining: the isomerization and the cracking of hydrocarbons ${ }^{1}$. Depending on the topology of the zeolite used and the selectivity it imparts, isomerization and cracking reactions form desired products by converting simple $n$-alkanes into various branched isomers and cleaving large hydrocarbons into smaller ones, respectively ${ }^{2}$. The majority of the constituents of many everyday substances, from gasoline to a plastic PET bottle, will thus have seen the inside of a zeolite catalyst and experienced the effect of shape selectivity ${ }^{3}$. Yet, despite this enormous economic importance of shape selectivity, we have only recently gained the insights needed to fully understand the molecular mechanisms that give rise to it.

This review focuses on hydroconversion reactions that proceed in the presence of excess hydrogen gas because they are relatively well understood and exemplify the recent improvements in understanding of shape selectivity. In hydroconversion reactions the acid sites inside the zeolite pores catalyse two competing reactions upon hydrocarbon exposure: hydroisomerization and hydrocracking. The hydroisomerization reactions convert linear hydrocarbons ( $n$ alkanes) into branched isomers; these can then be converted further through transfer of the branch along the molecular backbone, or through a second hydroisomerization reaction to form a di-branched isomer. Hydrocracking reactions break a hydrocarbon reactant into two smaller molecules, and proceed particularly easily if hydroisomerization has formed a so-called hydrocracking precursor: a molecule with two branches that are attached to the same carbon atom or to neighbouring carbon atoms. The detailed mechanism of the elementary hydrocarbon hydroconversion steps is well understood ${ }^{4}$. However, the large number of possible reactions and the fact that many molecules may act as intermediates or end up as products typically results in a complex distribution of product molecules that is not simple to predict.

Figure 1 illustrates hydroisomerization and hydrocracking reactions for a simple starting material (or 'feed') of pure decane. Suppose we carry out a hydrocracking reaction in sulphuric acid or with unstructured (amorphous) aluminosilicates. Because the (gasphase) free energies of formation of the various decane isomers shown are nearly identical, the product distribution after cracking 

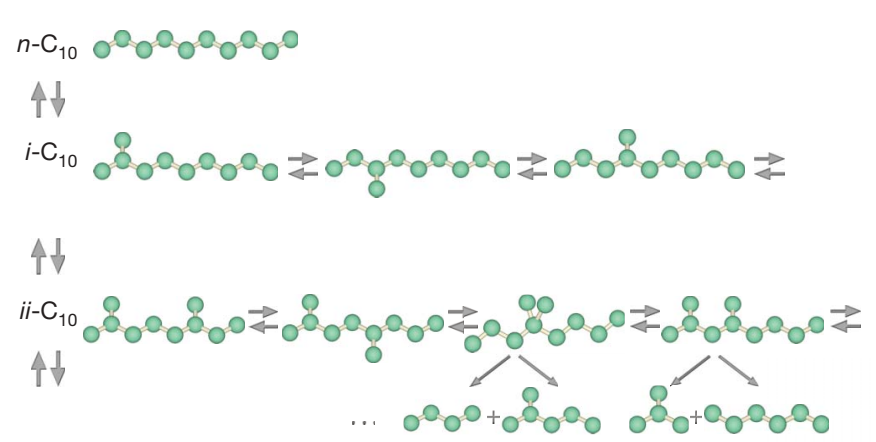

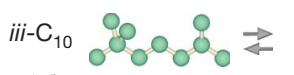
$4 \downarrow$

Figure 1 | Hydroisomerization and hydrocracking of $\boldsymbol{n}$-decane. The scheme illustrates some of the chemical reactions that can take place inside the pores of a zeolite. These include hydroisomerization reactions that convert $n$-decane $\left(n-\mathrm{C}_{10}\right)$ into its mono-branched $\left(i-\mathrm{C}_{10}\right)$, di-branched $\left(i i-\mathrm{C}_{10}\right)$, tribranched $\left(\right.$ iii- $\left.\mathrm{C}_{10}\right) \ldots$ isomers. A hydrocracking precursor is formed when hydroisomerization yields a molecule with two branches attached to the same or to next-neighbouring carbon atoms. Precursors with two branches hydrocrack relatively easily into a smaller linear and branched alkane. These isomers or their cracking products either continue to react or leave the zeolite as part of the product distribution.

will reflect the probability with which particular cracking precursors form. If all isomers form with equal probability, then simple statistical arguments ${ }^{5}$ predict that the highest number of reaction paths lead to hydrocracking precursors that have methyl groups close to the centre of the molecule. As illustrated in Fig. 1, such a molecule will be cleaved or 'cracked' between the two methyl groups. The net result is that the product molecules have a gaussian size distribution centred on $\mathrm{C}_{5}$, that is, on half the length of the feed molecule. Such ideal product distributions simply reflect the statistical probability of forming intermediates and product molecules and are obtained with hydroprocessing catalysts lacking shape selectivity. Zeolite catalysts that give rise to products deviating from the (statistically determined) ideal distribution exhibit shape selectivity. Here we focus on the simplest of cracking reactions involving the metal-catalysed activation of feed molecules through hydrogen $\left(\mathrm{H}_{2}\right)$ abstraction and the metal-catalysed deactivation of product molecules through hydrogen addition. When this metal functionality is absent (as in catalytic cracking) or defective, then hydrogen subtraction and addition can occur through several different pathways so that the reaction network becomes more complex. In such situations, it becomes commensurately more difficult to identify shape selectivity unambiguously ${ }^{51}$.

The original explanation ${ }^{6}$ for the shape selectivity associated with zeolite catalysis is simple and intuitive: the pores, or rather the active sites within the pores, exclusively process the molecules that fit inside. Yet in many instances, this picture cannot explain the mixtures of product molecules (the so-called product distributions) obtained in actual zeolite catalysis experiments and new forms of shape selectivity have been discovered (see Box 1) 7,8 $^{7,}$ For a more complete understanding of shape selectivity, we need to understand the effect of confinement on the various kinetic and thermodynamic effects that can influence the outcome of a zeolite-catalysed reaction. We show here that this requirement equates, in essence, to understanding the effect of confinement on adsorption and diffusion.

Significant technical advances over the last decades have made it possible to synthesize and characterize well-defined zeolite crystals, and to measure diffusion and adsorption processes accurately in a number of zeolite/hydrocarbon systems. But it is still not possible to obtain reliable experimental thermodynamic and kinetic data on a catalytic system under operating conditions. Here we argue that molecular simulation capabilities can now provide reliable thermodynamic and transport data, and that this capability enables systematic analyses of possible mechanistic explanations for experimentally observed product distributions. Such analyses, in turn, point to a general concept that explains the different types of shape selectivity seen in a wide range of catalytic systems. We show that this general shape selectivity concept not only explains known data and behaviour, it can also serve as a prognostic tool for the simulationbased discovery of those zeolite structures that are best suited for delivering the product distribution desired in a 'real' industrial hydrocarbon processing step.

\section{Simulating molecules in zeolites}

Although recent years have seen much progress in our ability to probe and image single molecules directly, it is at present still impossible to monitor directly how individual molecules move and react inside the pores of a zeolite under operating conditions. But these processes can be simulated on a computer. For such a simulation to deliver realistic results, it needs to use an accurate potential that correctly, and as quantitatively as possible, describes the interactions between the molecules that are present in the zeolite and between those molecules and the zeolite itself. If useful thermodynamic and transport properties are to be extracted, the simulation needs to run long enough for the system to explore the huge number of available configurations; that is, the simulation must 'sample' a sufficient number of different configurations to permit a meaningful statistical description of the results.

One of the first molecular dynamics simulations of molecules adsorbed in zeolites was published by Thomas and co-workers nearly 20 years ago 9 . Like other early work, it focused on the adsorption thermodynamics and diffusion of small molecules such as methane. But to be pertinent to our understanding of industrial zeolite catalysis, simulations need to investigate not small molecules like methane but long-chain hydrocarbons-an impossible task 20 years ago, simply because simulating long-chain hydrocarbon dynamics in zeolites would have required many millions of years of computer processor time. Their slow dynamics arises from the relatively small diffusion coefficients of long-chain hydrocarbons: they can be orders of magnitude smaller than that of methane, so the molecular dynamics simulations need to be commensurately longer ${ }^{10}$ to ensure that the molecules have diffused sufficiently far away from their initial position to generate new and statistically independent configurations and to provide meaningful sampling.

The challenge of how to simulate slowly diffusing molecules can be addressed by considering that such molecules are mostly trapped inside cage-like pore structures or at adsorption sites. Infrequent hopping to a neighbouring cage or adsorption site occurs only after a molecule has overcome a considerable free-energy barrier to diffusion. Such situations can be dealt with by exploiting the stochastic nature of Monte Carlo methods when inserting molecules in a zeolite: random insertions will occasionally occur at positions that correspond to the top of the free-energy barrier to diffusion, and the subsequent evolution of the system can then be simulated. This approach deals successfully with the computational problems caused by large diffusion barriers. But conventional Monte Carlo methods can efficiently insert only simple molecules like methane. In the case of long-chain molecules, billions of configurations would need to be generated to find one in which none of the hydrocarbon atoms overlaps with the zeolite or any of the other molecules included in the system. This difficulty can be overcome with an intelligent growing scheme that locates empty spots in the zeolite and then 'grows' the molecule. The most advanced simulation techniques combine these methods for dealing with diffusion and insertion problems and thus make it possible efficiently to simulate the behaviour of long-chain hydrocarbons in zeolites. One of the most successful techniques is configurational-bias Monte Carlo (CBMC). Depending on the simulation conditions used and the size of the inserted molecule, CBMC 


\section{Box 1 | Different forms of shape selectivity}

The historical definition of shape selectivity is that the product distribution will deviate from the ideal distribution if the formation of some of the molecules in Fig. 1 is inhibited by the constraints on molecular size and shape imposed by the pores. Besides exclusion due to the size of the molecules, the Box 1 figure provides a schematic illustration of the three additional effects that determine whether or not a particular product molecule will be formed with a zeolite catalyst of a given pore shape. In the most general case of reactant shape selectivity (Box 1 Figure a), one has a multi-component feed and only those molecules that are adsorbed by the zeolite and diffuse sufficiently fast to the active site will be converted. But the feed molecules not only need to reach the active site, they also have to be converted and the product molecules then need to diffuse away and finally desorb from the zeolite. The shape of the zeolite may influence each of these steps such that the product distribution changes. If a change in product distribution arises from the actual product formation step, the effect is known as transition state shape selectivity. Such a case is illustrated in Box 1 Figure b, where the shape of the zeolite pore inhibits the formation of a bulky (di-branched) molecule that is too big to fit inside. But transition state shape selectivity can also result in inverse shape selectivity: if the bulky product molecule being formed fits the zeolite pore optimally, it will be stabilized and hence can be formed preferentially over other products. Finally, Box 1 Figure c illustrates product shape selectivity, where diffusion limitations prohibit desorption of product molecules that are too large.

Consideration of these three basic effects underlying shape selectivity can give a good indication of which product molecules are likely to form. But for more reliable-ideally quantitative-predictions, we also need to take into account thermodynamic effects. For example, of all possible products, the molecule(s) with the lowest free energy of formation in the adsorbed phase will be preferentially formed (transition state shape selectivity) and the molecule(s) with the highest free energy of adsorption will preferentially desorb and accumulate in the product slate (product shape selectivity). Similarly, of all the reactants that can fit inside a zeolite catalyst, those with the lowest free energy of adsorption will be preferentially adsorbed and can then undergo reaction (reactant shape selectivity).

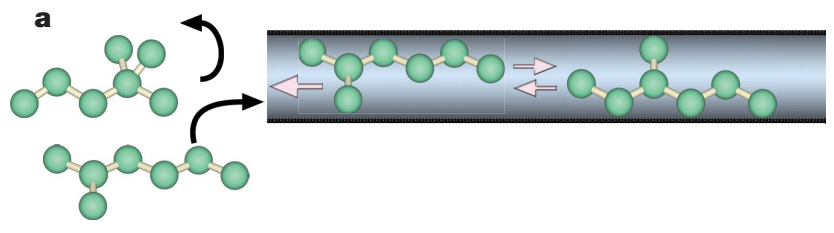

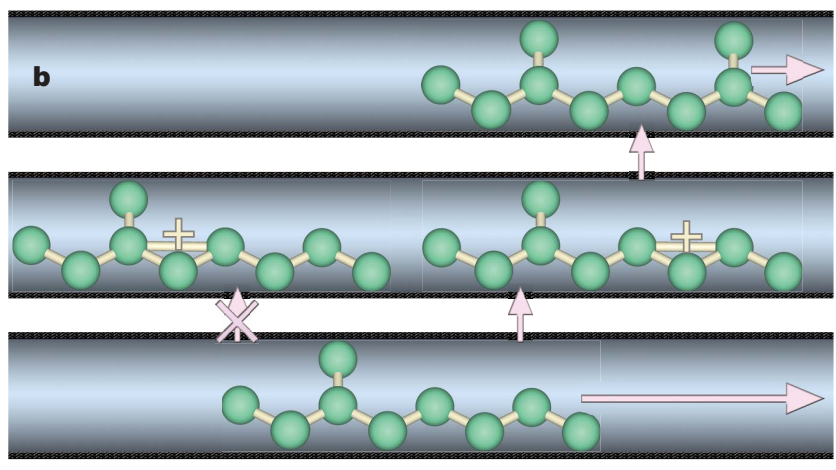

Box 1 Figure | Basic mechanisms giving rise to shape selectivity.

a, Reactant shape selectivity: molecules that are too large to enter the zeolite pores cannot reach acid sites for reaction and are therefore not converted into products. $\mathbf{b}$, Transition state shape selectivity: molecules

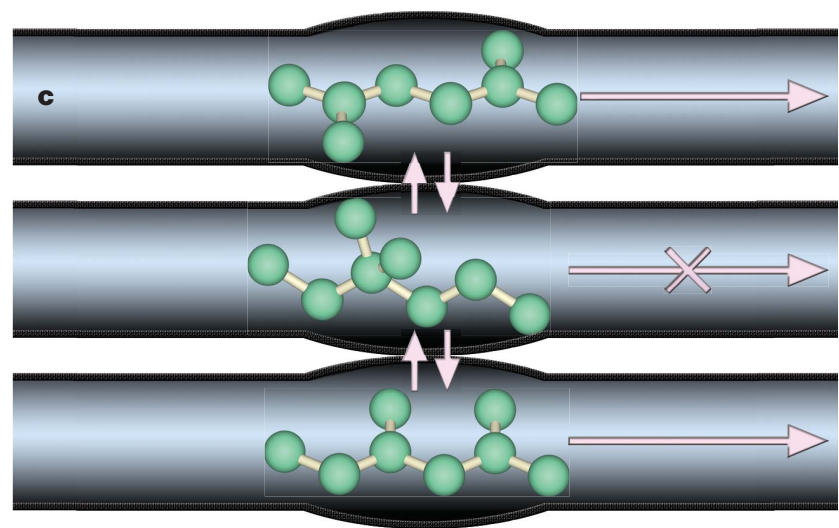

(and transition states) that are too large to fit inside a pore do not form. c, Product shape selectivity: new molecules are formed in the adsorbed phase, but are too large to desorb as a product. can be 10 to 40 orders of magnitude more efficient than conventional techniques ${ }^{11}$. Box 2 gives a more detailed description of this method and outlines how it can be used to obtain accurate adsorption isotherms ${ }^{12}$, free energies of formation of molecules residing inside zeolite pores, and diffusion coefficients ${ }^{13,14}$.

\section{The free-energy landscape}

As just discussed, simulations can provide us with reliable thermodynamic and kinetic data characterizing the adsorption and diffusion of organic molecules within a zeolite. This allows us to illustrate and validate the key concept we wish to present here, the 'free-energy landscape approach' to a molecular understanding of shape selectivity in zeolite catalysis. A central premise of this approach is that by ignoring the detailed chemical characteristics of a zeolite and simply quantifying instead how its topology affects the free energies of formation of the various reactants, intermediates and products involved (that is, the free-energy landscape of the reacting system), we can identify the fundamental interactions and processes that control the shape selectivity of a particular transformation.

We focus our discussions on hydroconversion reactions to make this point, but emphasize that the 'free-energy landscape approach' has some important limitations. Most importantly, it can be applied in a straightforward fashion only to simple reactions that occur at a single reaction site; if reaction pathways are more complex (an example being competing reactions occurring at single active sites or at pairs of such sites), then our simple approach may no longer apply. We also note that zeolites obviously catalyse many chemical reactions other than hydroconversion, and it is well known that different reaction classes are often efficiently catalysed by zeolites that differ in their basic chemical composition. For example, whereas hydroconversion requires aluminosilicates, shape selective oxidation reactions are in general catalysed by titanosilicates (zeolites in which the framework $\mathrm{Si}^{4+}$ is replaced by $\mathrm{Ti}^{4+}$ rather than $\mathrm{Al}^{3+}$ ). The chemical characteristics of zeolites thus clearly play an important role in achieving the desired catalytic activity. But within each general class 
Box 2 Simulating molecules in zeolites

The starting point of the simulation is the known crystal structure of the zeolite $^{37}$, from which we can generate the positions of the Si and O atoms. In our simulations we use periodic boundary conditions such that we mimic a perfect, infinitely large crystal without an external surface. As in most simulations studies, the zeolite is assumed to be rigid. Comparison of the results for flexible and rigid zeolites shows that flexibility has very little effect on the thermodynamic properties ${ }^{38}$, but might be more significant for the transport properties because flexibility may increase or decrease the free energy barrier for diffusion ${ }^{39}$. Even for diffusion these effects are too small and experiments are not sufficiently accurate to assess the conditions under which the assumption of a rigid zeolite structure may break down.

\section{Configurational-bias Monte Carlo}

Adsorption isotherms, which indicate the number of adsorbed molecules as a function of the pressure (chemical potential) of the gas in contact with the zeolite, are readily obtained from a Monte Carlo simulation in the grand-canonical ensemble in which temperature and chemical potential are imposed and the number of adsorbed molecules is a result of the simulation ${ }^{40}$. To change the number of adsorbed molecules, one of the Monte Carlo moves involves an attempt to add or remove a molecule. Such a move is subsequently accepted or rejected with a probability that depends on whether it has a favourable energy. Adding a molecule at a random position in the zeolite will only result in an acceptable conformation if it does not overlap with one of the atoms of the zeolite. For methane this is relatively easy and may only need the generation of, say, ten positions before an empty spot is found. Ethane requires two empty spots and so the number of attempts will be of the order of a hundred. Clearly, for the long-chain hydrocarbons of interest for catalytic applications the probability of generating a configuration in which none of the atoms overlaps is prohibitively small.

The CBMC technique has been developed ${ }^{41,42}$ to make the insertion of these long-chain molecules possible. In a CBMC simulation a molecule is not inserted at random but grown atom by atom using a method based on an algorithm developed by Rosenbluth and Rosenbluth ${ }^{43}$. During the growing step, overlap with the zeolite atoms is avoided by selecting (from the set of possible positions at which to add the next atom) the position with the lowest energy with the highest probability of acceptance. The conventional Monte Carlo scheme, however, relies on randomly generated configuration and hence the bias in the growing scheme towards configurations with the lowest energy would result in a scheme that would generate configurations that do not have a proper Boltzmann distribution. The key aspect in a CBMC simulation is that we compute the bias introduced in the growing step and this information is used in an acceptance rule that exactly removes this bias ${ }^{42}$; that is, the product of the probabilities of growing a particular configuration and of acceptance recovers the correct Boltzmann distribution.

The CBMC can be used in various ensembles. Combined with the grand-canonical ensemble, it allows for the insertion and deletion of long-chain hydrocarbons and hence the computation of a complete adsorption isotherm. CBMC allows us also to compute free energies. For small molecules, free energies are conveniently computed using the Widom test particle insertion method ${ }^{40}$. In this method, the free energy is expressed as an ensemble average related to the Boltzmann factor of the energy $\mathrm{U}^{+}$of randomly inserted test particles that probe the energy, but do not participate in the simulation: $\left\langle\exp \left(-U^{+} / k_{\mathrm{B}} T\right)\right\rangle$. For chain molecules, random insertion results in configurations that almost always overlap and hence have zero contribution to the ensemble average. Here, the CBMC scheme also allows the generation of biased configurations that do not overlap and hence have a non-zero contribution to this ensemble average. Also, for the free energy it is important to correct for the bias in the growing scheme ${ }^{37}$. Intermolecular potentials

For adsorption of hydrocarbons in zeolites it is convenient to use a united-atom description, in which the $\mathrm{CH}_{\mathrm{i}}$ groups are seen as one united atom. The united atoms are connected with bond-bending, bond vibrations and torsion potentials such that an accurate description of the internal structure of a hydrocarbon is made. The non-bonded, van der Waals interactions are optimized to reproduce the vapour-liquid phase behaviour of the alkanes ${ }^{44,45}$. The interactions of the $\mathrm{Si}, \mathrm{O}$ and $\mathrm{Al}$ atoms of the zeolite with the united atoms of the hydrocarbon are described with Lennard-Jones parameters ${ }^{46}$. These parameters have been optimized to accurately reproduce the steps that observed in the experimental adsorption isotherms ${ }^{46}$.
Adsorption isotherms and diffusion coefficients

Typical examples of the accuracy that can now be achieved for computing thermodynamic and transport data are shown in Box 2 Figure $a$ and $b$, respectively. The intermolecular interactions have been optimized for different molecules and/or materials and therefore the results shown in these figures demonstrate that one can predict adsorption isotherms ${ }^{46}$ and diffusion coefficients ${ }^{14}$ of long-chain hydrocarbons in various zeolites. Molecular simulation studies have predicted many different phenomena, such as commensurate freezing ${ }^{12}$ and entropic separation ${ }^{47}$, that were only recently confirmed by experiments ${ }^{48,49}$.

Rare events simulations

A very small diffusion coefficient often is the result of molecules that are trapped in low (free) energy sites and only once in a while hop to another adsorption site by crossing a free-energy barrier that separates these two sites. In a rare event simulation this hopping rate is computed in two steps; first, the probability that a molecule can be found on top of the barrier, followed by a separate simulation in which the probability is computed so that this molecule actually reaches the other adsorption site ${ }^{40}$. The probability of finding a molecule on top of the barrier can be computed directly from the free-energy profile, which is the free energy as a function of the position of the molecule in the zeolite. The second step involves a large number of very short molecular dynamics simulations in which a molecule is initialized on top of this barrier and the probability is determined that this molecule does end up in the neighbouring adsorption site and does not return to its original location. As this involves a simulation that starts on top of the barrier, it is much faster than the time it takes a molecule to 'climb' this barrier. These rare event methods have been applied to zeolites at low $^{14}$ (see Box 2 Figure b) and even high loadings ${ }^{13}$

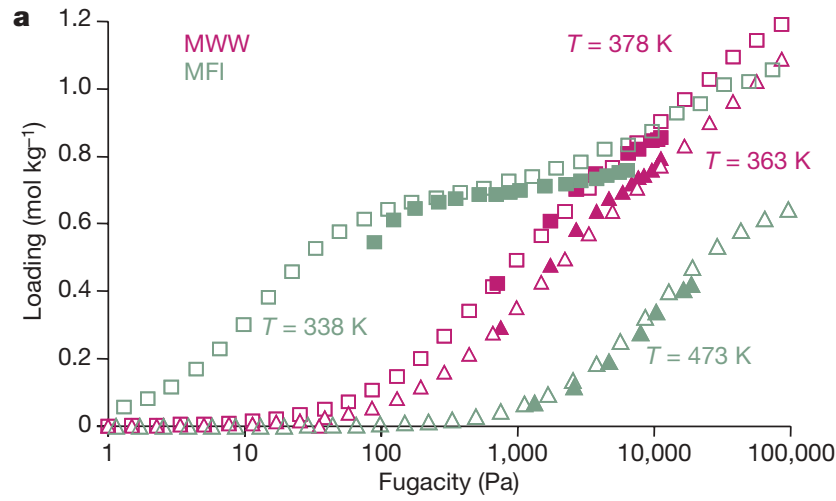

b

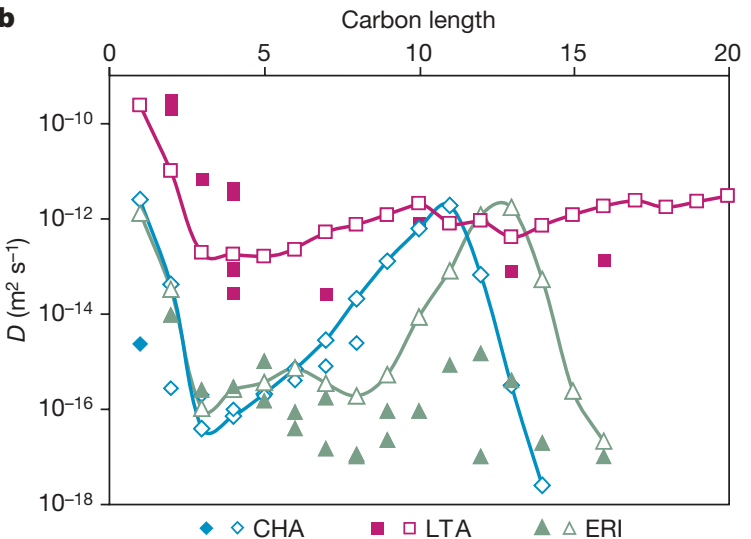

Box 2 Figure | Thermodynamic and kinetic data from molecular simulations. Typical examples of the application of molecular simulation; the adsorption isotherms (a) have been obtained via CBMC simulations and the diffusion coefficients (b) by rare events simulations. Comparison with the experimental data (filled symbols) illustrates the agreement that can be obtained. a, The adsorption isotherms give the loading of $n$-hexane in MWW and MFI as a function of the fugacity (pressure) at different temperatures (data from ref. 50). b, Diffusion coefficients $D$ of the $n$-alkanes in the CHA, LTA and ERI as a function of chain length (data from ref. 14). 
of reactions (for example, hydroconversion, or hydrocarbon oxidation), the free-energy landscape approach to understanding shape selectivity should hold; that is, among all zeolites with a chemical composition appropriate for a particular hydrocarbon conversion process of interest, this approach can be applied to identify those zeolites that have a topology optimally suited to generating the maximum yield of the desired product(s).

The zeolite-catalysed conversion of $n$-decane illustrates the freeenergy landscape approach. This conversion involves many competing reactions (illustrated in Fig. 1), and a first step in identifying the preferred reaction path(s) in such a complex system-and hence the dominant product(s) - is the quantification of the system's freeenergy landscape, and in particular how this landscape changes with zeolite topology. The challenge here is that although the gas-phase free energies of formation for most molecules in the reaction scheme of Fig. 1 are known and similar, the free energies of formation of the molecules when present in the adsorbed phase in a zeolite are rarely known. Exceptions are molecules that cannot react because they do not fit inside a zeolite pore; they exhibit a prohibitively large positive free energy of formation. In the case of zeolites with very wide pores, adsorbed molecules will be in physical equilibrium with the gas phase and will probably be unaffected by condensed-phase thermodynamic constraints. But when the fit becomes snugger, molecules formed inside the zeolite may no longer be able to desorb as products and products that have left the zeolite may no longer be able to re-adsorb; that is, molecules are locked in or locked out. Under such conditions, the gas phase and adsorbed phase can no longer equilibrate and the free-energy landscape imposed by the zeolite topology on the reacting system will leave its signature on the product distribution. Such lack of equilibration between gas phase and adsorbed phase is endemic to larger molecules in industrial processes such as we consider here. We note that even though complete equilibrium will never be achieved (as in almost all processes), almost all thermodynamic arguments intrinsically assume equilibration. In the context of the present discussion, we argue that despite the lack of full equilibration, a quantification of the adsorbed-phase free-energy landscape nevertheless serves as a useful starting point that can help us to arrive at a quantitative description of zeolite catalysis.

Quantification of the free-energy landscape associated with a particular zeolite topology and particular hydrocarbons has long been impossible, but can now be achieved using sophisticated simulation methods (see Box 2). We are accordingly able to compile data such as are shown in Fig. 2a, which illustrate how the free energy of formation of five intermediates involved in $n$-decane hydroconversion changes relative to that of $n$-decane as a function of zeolite structure. We note that the relative adsorption coefficients of the five intermediates adsorbed on the zeolite pore walls are a measure of the ease with which the individual intermediates form (relative to $n$-decane), and not of their proton affinity ${ }^{4}$ or intrinsic reactivity. Compared with the corresponding gas-phase values, which are nearly identical for all isomers, FAU (a zeolite with large cages) has little effect on the relative free energy of formation of all five molecules investigated. By contrast, the zeolite TON (with narrow channels) makes a prohibitively high and positive contribution to the free energy of formation of di- and tri-branched $n$-decane isomers. This effect quantitatively confirms the traditional concept of shape selectivity, which predicts that di- and tri-branched isomers will not form because they are too large for the TON pores.

But a comparison of $n$-decane conversion in the zeolites MFI and MEL illustrates the limitations of the traditional shape selectivity concept and the importance of quantitative free-energy data: despite their similar structures, the zeolites generate markedly different product distributions ${ }^{15}$. Both zeolites can accommodate all the molecules involved in $n$-decane conversion that are shown in Fig. 1, and these may desorb after their formation as final products or serve as reaction intermediates and react further. The probability of a particular molecule forming during a zeolite-catalysed process is directly proportional to its free energy of formation in the adsorbed phase. In the case of $n$-decane conversion, this free energy is dominated by the zeolite contribution unless zeolites with very wide pores such as FAU are used (as discussed above).

The data in Fig. 2 illustrating this contribution for key reaction intermediates ${ }^{16}$ clearly indicate that 4,4-dimethyl octane is the most stable species in MFI whereas 2,4-dimethyl octane is the most stable
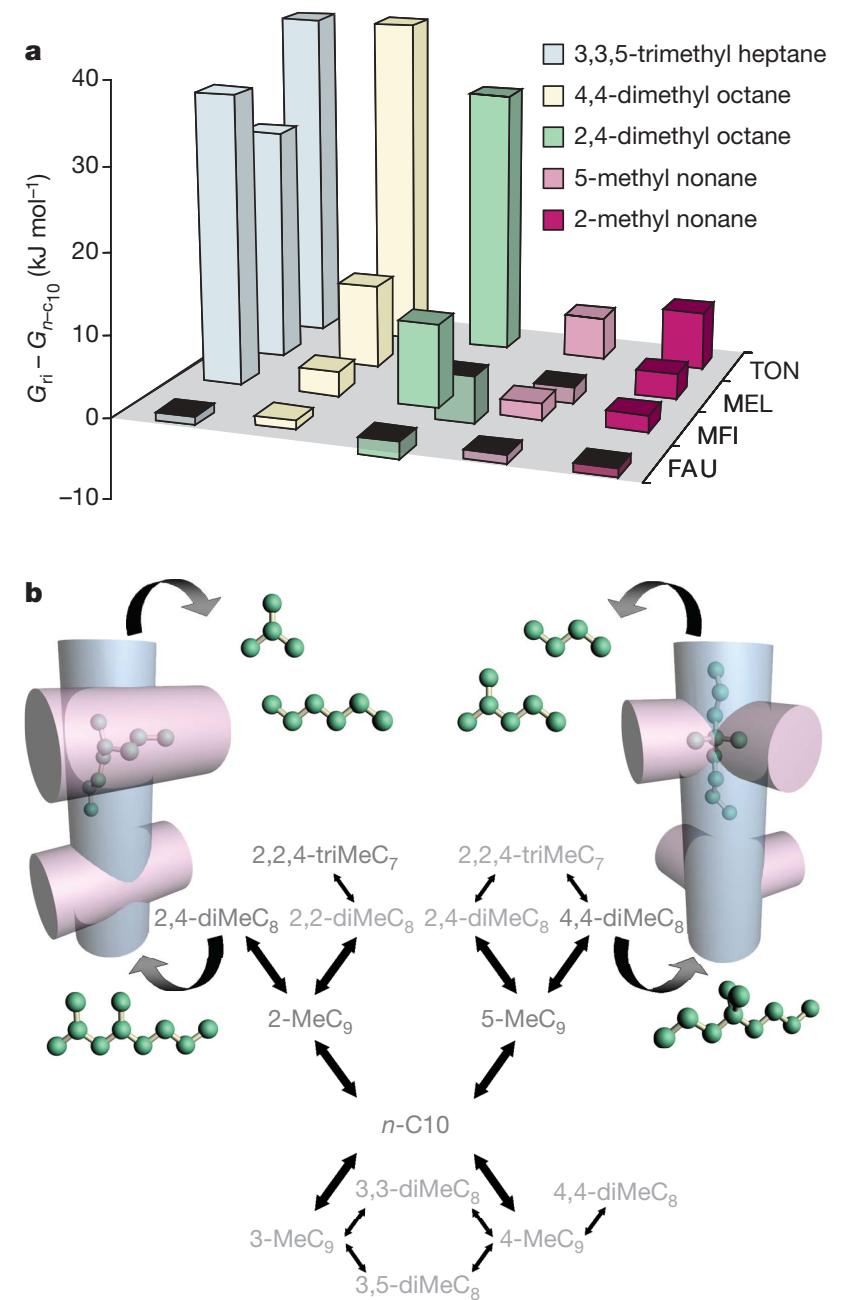

Figure 2 | Schematic representation of the free-energy model. The contribution of the zeolite topology to the free energy of formation of key reaction intermediates of the hydroconversion of $n$-decane in different zeolite structures (data taken from ref. 16). a, The free-energy differences between the reaction intermediates and $n$-decane for different types of zeolites: $G_{\mathrm{ri}}-G_{n-C_{10}}$. TON is a small-pore zeolite that greatly increases the free-energy differences (for the tri- and di-branched isomers the free energies are 95.8 and $123.6 \mathrm{~kJ} \mathrm{~mol}^{-1}$, respectively, off the scale of the figure). FAU is a large-pore zeolite that contributes little to the free-energy differences. MEL and MFI have similar structures to each other but with pore widths intermediate between those of TON and FAU. The contributions of MEL and MFI are similar for most reaction intermediates, but there are marked differences in free energies for some specific reaction intermediates. The consequences for the reactions in the pores are illustrated in b. MFI (the structure on the right) prefers to form 4,4-dimethyl octane because it is commensurate with the zig-zag channel and hence forms a nice fit (see the molecule in the zeolite), whereas MEL (the structure on the left) prefers to form 2,4-dimethyl octane, which snugly fits in the larger intersection. As a consequence, the reaction paths in MFI are dominated by the path $n-\mathrm{C}_{10} \rightarrow 5-\mathrm{MeC}_{9} \rightarrow 4,4-\mathrm{MeC}_{8}$, whereas in MEL the dominant path is: $n-\mathrm{C}_{10} \rightarrow 5-\mathrm{MeC}_{9} \rightarrow 2,4-\mathrm{MeC}_{8}$. The reaction scheme shown in Fig. 1 shows that 2,4- $\mathrm{MeC}_{8}$ and 4,4- $\mathrm{MeC}_{8}$ are cracking precursors that yield branched and linear butane, respectively. This explains why MEL produces more isobutane relative to butane and why MFI exhibits the opposite trend in the experimental product distribution ${ }^{15}$. 
species in MEL. The dominant reaction path involving di-branched isomers thus proceeds through two different intermediates in MFI and MEL (4,4-dimethyloctane and 2,4-dimethyloctane, respectively). Rare event simulations of these systems have shown ${ }^{17}$ that the free-energy barriers for the hopping of both intermediates from one pore intersection to another are so high that the diffusion coefficients are impossibly small; in other words, neither molecule can leave after it has been produced inside the zeolite, so that equilibration with the gas-phase cannot be reached. Desorption of either hydrocarbon is only possible after cracking converts 4,4-dimethyl octane into $n$-butane product in the case of MEL, or 2,4-dimethyl octane into isobutane product in the case of MFI. This comparative example illustrates how zeolites can control product distributions by favouring the formation of particular reaction intermediates. We also note that both reaction intermediates are typical 'ship-in-a-bottle' molecules that can form inside a zeolite but not desorb. Any attempt to experimentally determine the adsorbed-phase free energies of such molecules is a major challenge. Simulations may thus be the only viable means for obtaining the information that is needed to develop a mechanistic explanation for the different catalytic properties of topologically similar zeolites such as MEL and MFI.

The zeolite-catalysed hydroconversion of $n$-hexadecane $\left(n-\mathrm{C}_{16}\right)$ provides another illustration of the insights to be gained from the use of simulations and quantitative free-energy considerations. Empirical evidence has taught us that to maximize the production of molecules with the highest octane number (that is, di-branched hexane $\left(\mathrm{C}_{6}\right)$ isomers) and to minimize the formation of low-octane hexane isomers (especially $n$-hexane), the conversion needs to be carried out in the presence of zeolite catalysts with one-dimensional, tube-like pores ${ }^{18}$. Interestingly, the free energies of formation of the molecules involved in the conversion suggest that at very low reactant concentrations, the selectivity of the process changes little with the pore width of the zeolites used ${ }^{17}$. This observation is at variance with experimental results. However, the actual process is carried out at high pressures where the zeolites are completely filled, and calculations carried out under these conditions predict product selectivities that agree with observed product distributions. In this case, the pressure-dependence of the simulation results signals that the selectivity of the process is an entropic effect: the high operating pressure favours formation of those $\mathrm{C}_{6}$ isomers that can optimally pack inside the zeolite ${ }^{19}$. Subsequent desorption of $\mathrm{C}_{6}$, followed by readsorption and further reaction could in principle obscure this effect. But the long $\mathrm{C}_{16}$ reactant molecules block any re-adsorption within the filled zeolite pores and prevent equilibration with the gas phase, thereby ensuring that the relative abundance of the various $\mathrm{C}_{6}$ products leaving the reactor bears the signature of the relative entropy of the $\mathrm{C}_{6}$ isomers in the adsorbed phase. As a result, the selectivity of the $n$-hexadecane $\left(n-\mathrm{C}_{16}\right)$ hydroconversion process can be optimized by using zeolite structures with pore diameters that allow for the most efficient packing of branched $\mathrm{C}_{6}$ molecules.

The above examples illustrate that quantitative thermodynamic and kinetic data are essential to move from intuitive yet speculative explanations for shape selectivity to a firm mechanistic understanding. In fact, several shape-selective transformations catalysed by zeolites have been re-analysed using the principles we have outlined above, resulting in the identification of selectivity mechanisms different from those proposed originally ${ }^{19-23}$.

\section{In silico screening}

As illustrated in the preceding section, computer simulations are now at a stage where they can accurately quantify the free-energy landscape imposed by a given zeolite topology on a reacting system and thus help us to develop mechanistic explanations for why a reaction of interest yields the product distribution that is experimentally observed. But a more challenging question is whether this simulation-based methodology has predictive power; that is, whether it can screen zeolite structures to identify those particularly well suited for new applications. A case in point is hydrodewaxing, an important refining process that converts the longest hydrocarbons present in a fuel or lubricant feed into smaller molecules and thus eliminates the risk of wax precipitation and associated engine problems during later use. In hydrodewaxing, the zeolite catalyst thus needs to convert the longest hydrocarbons while leaving shorter hydrocarbons unharmed. Expressed in terms of free energies of formation, the zeolite should have a topology that maximizes the difference between the free energies of formation of the molecule to be converted, say $n-C_{25}$, and a reference molecule that needs to remain untouched, such as $n-\mathrm{C}_{10}$.

The result of such 'screening by computer' (summarized in Fig. 3) shows that the optimally performing zeolites are ZSM-48, MTW, GON, SFE and OFF. These zeolites all have pores with a typical tubular character, and pore diameters that are optimum for absorbing the long wax molecules that need to be converted during hydrodewaxing. The use of zeolites SFE and OFF in this context has been explored ${ }^{24,25}$ but not pursued owing to practical difficulties with synthesis, whereas ZSM-48 and MTW are at the heart of intellectual property activity ${ }^{26-28}$ on hydrodewaxing applications. Interestingly, GON had not yet been considered in this context before the computer screening, but a patent application has now been filed for a dewaxing process based on $\mathrm{GON}^{29}$. Similarly, computer screening also identified STI for dewaxing ${ }^{52}$.That patent applications can be entirely based on molecular simulations illustrates the considerable progress that has been made in this field since Thomas and coworkers carried out the first simulation on hydrocarbon behaviour in a zeolite.

\section{Simplification to success}

We believe that the explanation and prediction of shape selectivity on the basis of a quantitative free-energy landscape is a widely applicable and useful approach for understanding zeolite catalysis. But it is based on some important assumptions that should be kept in mind. As mentioned before, the simulations we have discussed all assume that once a particular family of zeolite with suitable chemical features has been identified as a promising type of catalyst for a given class of reactions, topology will control catalyst performance and the exact chemical composition of the zeolite will have only a minor influence. This allows us to compute and compare the free energies of

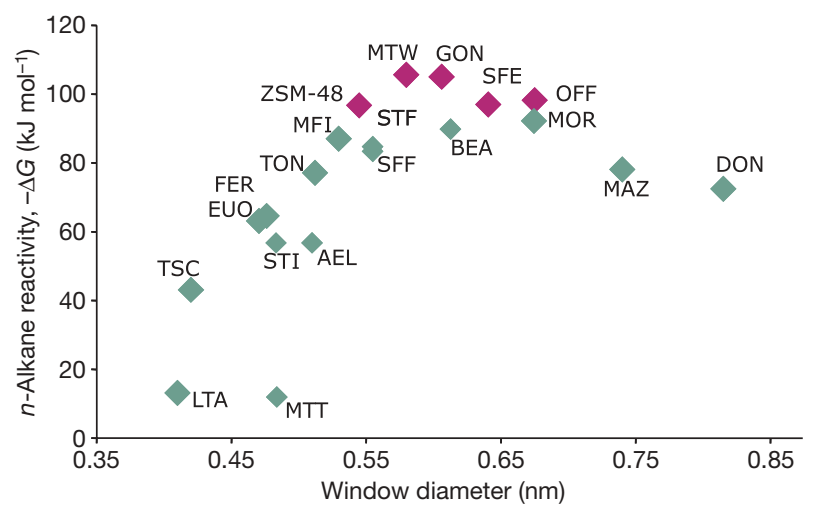

Figure 3 | Zeolite screening by computer. For hydrodewaxing, the optimal reactivity of a structure can be expressed in terms of the free energy of adsorption $\Delta G$ of a long $n$-alkane $\left(n-C_{25}\right)$. Frequently, zeolites are characterized by their window diameter, that is, the smallest diameter molecules 'see' when moving through the material. Topologies that exhibit a highly negative value preferentially convert long instead of short $n$-alkanes, that is, they optimally remove the waxes and leave the shorter hydrocarbons. Indirect experimental evidence for the validity of this thermodynamic approach is the match between the optimum structure and the focus of a recent flurry in patenting activity. The topologies in red have been patented by Shell ${ }^{26}$ and ExxonMobil ${ }^{27}$ and GON has recently been 'discovered' by simulations ${ }^{29}$. 
formation of reactants, intermediates and products in various silica zeolite structures without explicitly considering the chemical reactions as such. The fact that our approach is successful despite such a striking - to many readers, perhaps even alarming-simplification can be explained in part by the findings of very accurate quantum chemical calculations ${ }^{30,31}$ : detailed investigations of how zeolitecatalysed reactions proceed show that the nature of the acid site is remarkably similar for zeolites with markedly different topologies. That is, the acid sites in, say, narrow-pore or large-pore zeolites differ little in their reactivity.

The exact location of acid sites is known for a few zeolites and has been used when calculating the free energies of formation for a number of hydrocarbons in these zeolites ${ }^{32}$. The results support the assumption that the difference between the free energy of formation of different hydrocarbons in the same zeolite is almost unaffected by the exact location - and even the presence-of acid sites; that is, freeenergy differences depend almost exclusively on zeolite topology. All computations of free energies of formation are based on free-energy differences between the molecule that is formed and a reference molecule, so simulations aimed at determining free-energy differences can use all-silica structures as excellent approximations of acid zeolites and thus avoid the complex problem ${ }^{33}$ of having to account for the exact location — or at least the distribution — of acid sites.

Another important assumption underlying the free-energy landscape approach is that the Brønsted-Evans-Polanyi relationship applies. This relationship holds that the free-energy barrier that needs to be surmounted to form a given molecule from its starting material is proportional to the free energy of formation of that molecule. Put differently, the impact of a given zeolite topology on reaction kinetics mirrors its impact on the free energy of formation of the molecules that are formed. The Brønsted-Evans-Polanyi relationship is based on the simple idea that the transition state of a reaction largely resembles the product molecule, and is well known to be valid for a wide range of simple reactions such as hydroisomerization and hydrocracking that occur at single catalytic sites. But reaction pathways can be complex and, for example, require the involvement of more than one catalytic site. In such instances, the Brønsted-EvansPolanyi relationship will often break down and the free-energy landscape approach to probing zeolite catalysis will no longer be applicable. In this context, we also note that if the BrønstedEvans-Polanyi relationship does hold, the question of whether or not complete equilibrium is reached during reaction is not particularly important because the reaction kinetics is directly coupled to thermodynamics. This direct coupling means that quantitative information on the impact of the zeolite on the free energies of formation provide a reliable indication of which product molecules will form preferentially.

As the preceding discussion has clearly shown, the free-energy landscape approach to understanding - and even predicting - shape selectivity in zeolite catalysis is based on a number of drastic simplifications. But the success of the approach, as illustrated by the specific examples mentioned in this Review, emboldens us to conclude that a fairly simple but accurate and quantitative thermodynamic analysis will often suffice to explain in detail the product distribution characteristics of particular processes catalysed by a given zeolite. At present, the simulations that enable these thermodynamic analyses involve a significant idealization of industrial catalysis, for they do not consider catalysis occurring at the outside of zeolite crystals, nor zeolite crystal defects, stacking faults or intergrowths. But the fact that the simulated results compare well with experimental reference data obtained for nearly perfect zeolite crystals is encouraging. The next step will be systematically to include in simulations the effect of imperfections on the thermodynamic and transport properties, thus allowing us to describe not only ideal zeolite catalysts but also industrial zeolite-based catalysts. It should also be possible to expand the use of the free-energy landscape approach to zeolite-catalysed reactions other than hydroconversion reactions. Hydroconversions have been the focus of this Review for the simple yet practically important reason that a wealth of experimental data exists that documents shape selectivity for this reaction type; for most other reactions, shape selectivity has not been established unambiguously.

\section{To the future}

The approximately 180 zeolite structures known to exist constitute only a very small fraction of the more than 2.5 million structures that are feasible on theoretical grounds ${ }^{34}$. Such a database of hypothetical zeolite structures has been regarded an important step towards "designer catalysts" 35 , and it can in principle be screened for zeolites that are suitable for particular applications using the same methodology used to screen existing zeolites for their hydrodewaxing performance. To cope with such a large number of structures and to identify efficiently those with useful and superior catalytic properties will clearly involve enormous computational challenges. But even if effective screening is accomplished and successfully used to identify novel catalysts that allow us to use increasingly scarce fossil fuels more efficiently, any such in silico promise can only be realized if it is also possible to synthesize the identified structures. We expect that computer simulations will prove invaluable in this regard as well, by delivering increasingly detailed mechanistic insights into the nucleation and crystal growth of zeolites ${ }^{36}$ that might eventually allow us to rationally control and guide these processes such that they form desired new zeolite structures.

1. Corma, A. Inorganic solid acids and their use in acid-catalyzed hydrocarbon reactions. Chem. Rev. 95, 559-614 (1995).

2. Corma, A. From microporous to mesoporous molecular sieve materials and their use in catalysis. Chem. Rev. 97, 2373-2419 (1997).

3. Auerbach, S. M., Carrado, K. A. \& Dutta, P. K. (eds) Handbook of Zeolite Science and Technology (Marcel Dekker, New York, 2004).

4. van Santen, R. A. \& Neurock, M. Molecular Heterogeneous Catalysis: A Conceptual and Computational Approach (Wiley-VCH, Weinheim, 2006).

5. Froment, G. F. Kinetics of the hydroisomerization and hydrocracking of paraffins on a platinum containing bifunctional Y-zeolite. Catal. Today 1, 455-473 (1987).

6. Weisz, P. B. \& Frilette, V. J. Intracrystalline and molecular-shape-selective catalysis by zeolite salts. J. Phys. Chem. 64, 382 (1960).

7. Degnan, T. F. The implications of the fundamentals of shape selectivity for the development of catalysts for the petroleum and petrochemical industries. J. Catal. 216, 32-46 (2003)

8. Weitkamp, J., Ernst, S. \& Puppe, L. in Catalysis and Zeolites (eds Weitkamp, J. \& Puppe, L.) 327-376 (Springer, Berlin, 2001)

9. Yashonath, S., Thomas, J. M., Nowak, A. K. \& Cheetham, A. K. The siting, energetics and mobility of saturated hydrocarbons inside zeolitic cages: methane in zeolite Y. Nature 331, 601-604 (1988).

10. June, R. L., Bell, A. T. \& Theodorou, D. N. Molecular dynamics of butane and hexane in silicalite. J. Phys. Chem. 96, 1051-1060 (1992)

11. Smit, B. \& Siepmann, J. I. Simulating the adsorption of alkanes in zeolites. Science 264, 1118-1120 (1994).

12. Smit, B. \& Maesen, T. L. M. Commensurate 'freezing' of alkanes in the channels of a zeolite. Nature 374, 42-44 (1995).

13. Beerdsen, E., Smit, B. \& Dubbeldam, D. Molecular simulation of loading dependent slow diffusion in confined systems. Phys. Rev. Lett. 93, 248301 (2004).

14. Dubbeldam, D., Calero, S., Maesen, T. L. M. \& Smit, B. Incommensurate diffusion in confined systems. Phys. Rev. Lett. 90, 245901 (2003).

15. Jacobs, P. A., Martens, J. A., Weitkamp, J. \& Beyer, H. K. Shape-selectivity changes in high-silica zeolites. Faraday Discuss. Chem. Soc. 72, 353-369 (1981).

16. Schenk, M., Smit, B., Vlugt, T. J. H. \& Maesen, T. L. M. Shape selectivity in alkane hydroconversion. Angew. Chem. Int. Edn Engl. 40, 736-738 (2001).

17. Schenk, M. et al. Inverse shape selectivity revised. Angew. Chem. Int. Edn. Engl. 41, 2500-2502 (2002).

18. Santilli, D. S., Harris, T. V. \& Zones, S. I. Inverse shape selectivity in molecular sevies: Observations, modelling, and predictions. Microporous Mater. 1329-341 (1993).

19. Schenk, M. et al. Shape selectivity through entropy. J. Catal. 214, 88-99 (2003).

20. Calero, $\mathrm{S}$. et al. The selectivity of $\mathrm{n}$-hexane hydroconversion on MOR-, MAZ- and FAU-type zeolites. J. Catal. 228, 121-129 (2004).

21. Maesen, T. L. M., Calero, S., Schenk, M. \& Smit, B. Understanding cage effects in the n-alkane conversion on zeolites. J. Catal. 237, 278-290 (2006).

22. Maesen, Th. L. M. et al. The shape selectivity of paraffin hydroconversion on TON-, MTT- and AEL-type Sieves. J. Catal. 188, 403-412 (1999).

23. Dubbeldam, D., Calero, S., Maesen, T. L. M. \& Smit, B. Understanding the window effect in zeolite catalysis. Angew. Chem. Int. Edn Engl. 42, 3624-3626 (2003).

24. Rosenbaum, J. M. \& Howell, R. L. Dewaxing process. European Patent Application No. 1037956 (1999). 
25. Chen, N. Y., Schlenker, J. L., Garwood, W. E. \& Kokotailo, G. T. TMA-offretite. Relationship between structural and catalytic properties. J. Catal. 86, 24-31 (1984).

26. Duhoux, E. et al. Process to prepare a lubricating base oil and its use. European Patent Application No. 1791931 (2006).

27. Murphy, W. J. et al. Improved molecular sieve containing hydrodewaxing catalysts. US Patent Application No. 2006/0073962 (2006).

28. Benazzi, E., Guillon, E. \& Martens, Y. Catalyst and its use for improving the pour point of hydrocarbon feedstocks. European Patent Application No. 2004/ 0290680 (2004)

29. Maesen, T. L. M., Beerdsen, E. \& Smit, B. Dewaxing process using zeolites MTT and GON. US Patent Application No. $2007 / 0029229$ (2007).

30. Rozanska, X. et al. A periodic DFT study of isobutene chemisorption in protonexchanged zeolites: dependence of reactivity on the zeolite framework structure. J. Phys. Chem. B 107, 1309-1315 (2003).

31. Clark, L. A., Sierka, M. \& Sauer, J. Computational elucidation of the transition state shape selectivity phenomenon. J. Am. Chem. Soc. 126, 936-947 (2004).

32. Calero, S. et al. A coarse-graining approach for the proton complex in protonated aluminosilicates. J. Phys. Chem. B 110, 5838-5841 (2006).

33. Garcia-Perez, E. et al. A computational method to characterize framework aluminum in aluminosilicates. Angew. Chem. Int. Edn Engl. 46, 276-278 (2007).

34. Earl, D. J. \& Deem, M. W. Toward a database of hypothetical zeolite structures. Ind. Eng. Chem. Res. 45, 5449-5454 (2006).

35. Thomas, J. M. \& Klinowski, J. Systematic enumeration of microporous solids: towards designer catalysts. Angew. Chem. Int. Edn Engl. 46, 7160-7163 (2007).

36. Auerbach, S. M., Ford, M. H. \& Monson, P. A. New insights into zeolite formation from molecular modeling. Curr. Opin. Colloid Interf. Sci. 10, 220-225 (2005).

37. Baerlocher, Ch. \& McCusker, L. B. Database of Zeolite Structures /http://www.izastructure.org/databases/> (Structure Commission of the International Zeolite Association, IZA-SC).

38. Vlugt, T. J. H. \& Schenk, M. Influence of framework flexibility on the adsorption properties of hydrocarbons in the zeolite silicalite. J. Phys. Chem. B 106, 12757-12763 (2002).

39. Demontis, P. \& Suffritti, G. B. Structure and dynamics of zeolites investigated by molecular dynamics. Chem. Rev. 97, 2845-2878 (1997).
40. Frenkel, D. \& Smit, B. Understanding Molecular Simulations: From Algorithms to Applications 2nd edn (Academic Press, San Diego, 2002).

41. Siepmann, J. I. \& Frenkel, D. Configurational-bias Monte Carlo: A new sampling scheme for flexible chains. Mol. Phys. 75, 59-70 (1992).

42. Frenkel, D., Mooij, G. C. A. M. \& Smit, B. Novel scheme to study structural and thermal properties of continuously deformable molecules. J. Phys. Condens. Matter 4, 3053-3076 (1992).

43. Rosenbluth, M. N. \& Rosenbluth, A. W. Monte Carlo simulations of the average extension of molecular chains. J. Chem. Phys. 23, 356-359 (1955).

44. Siepmann, J. I., Karaborni, S. \& Smit, B. Simulating the critical properties of complex fluids. Nature 365, 330-332 (1993).

45. Siepmann, J. I., Martin, M. G., Mundy, C. J. \& Klein, M. L. Intermolecular potentials for branched alkanes and the vapour liquid equilibria of $n$-heptane, 2-methylhexane, and 3-ethylpentane. Mol. Phys. 90, 687-693 (1997).

46. Dubbeldam, D. et al. Force field parametrization through fitting on inflection points in isotherms. Phys. Rev. Lett. 93, 088302 (2004).

47. Krishna, R., Smit, B. \& Vlugt, T. J. H. Sorption-induced diffusion-selective separation of hydrocarbon isomers using silicalite. J. Phys. Chem. A 102, 7727-7730 (1998).

48. Morell, H. et al. Structural investigation of silicalite-I loaded with $\mathrm{n}$-hexane by $\mathrm{X}$-ray diffraction, Si-29 MAS NMR, and molecular modeling. Chem. Mater. 14, 2192-2198 (2002).

49. Yu, M., Falconer, J. L. \& Noble, R. D. Adsorption of liquid mixtures on silicalite-1 zeolite: A density-bottle method. Langmuir 21, 7390-7397 (2005).

50. Dubbeldam, D. et al. United atom force field for alkanes in nanoporus materials. Phys. Chem. B 108, 12301-12313 (2004).

51. Maesen, T. L. M., Calero, S., Schenk, M. \& Smit, B. Alkane hydrocracking: shape selectivity or kinetics? J. Catal. 221, 241-251 (2004).

52. Zones, S. I. et al. Hydrocarbon conversion using molecular sieve SSZ-75. US Patent Application 2007/0284284 (2007).

Acknowledgements We thank S. Calero, D. Dubbeldam, D. Frenkel, R. Krishna and M. Schenk. This work was supported by the EC through the Marie Curie EXT programme BiMaMoSi.

Author Information Reprints and permissions information is available at www.nature.com/reprints. Correspondence should be addressed to B.S. (berend-smit@berkeley.edu) or T.L.M.M. (tmaesen@chevron.com). 